\title{
Errors-In-Variables Anisotropic Extended Orthogonal Procrustes Analysis
}

\author{
Eleonora Maset, Fabio Crosilla, and Andrea Fusiello
}

\begin{abstract}
This paper presents a novel total least squares solution of the anisotropic row-scaling Procrustes problem. Ordinary least squares Procrustes approach finds the transformation parameters between origin and destination sets of observations minimizing errors affecting only the destination one. In this study, we introduce the Errors-In-Variables model in the anisotropic Procrustes analysis problem and present a solution that can deal with the uncertainty affecting both sets of observations. The algorithm is applied to solve the image exterior orientation problem. Experiments show that the proposed total least squares method leads to an accuracy in the parameters estimation that is higher than the one reached with the ordinary least squares anisotropic Procrustes solution when the number of points, whose coordinates are known in both the image and the external systems, is small.
\end{abstract}

Index Terms-Anisotropic Extended Orthogonal Procrustes Analysis (AEOPA), Errors-in-Variables (EIV) model, exterior orientation, Total Least Squares (TLS)

\section{INTRODUCTION}

$\mathbf{P}$ ROCRUSTES analysis is a well known technique used to directly provide least squares (LS) alignment among corresponding points belonging to a generic $k$-dimensional space, in order to satisfy their maximum agreement. It is particularly appealing from the computational point of view, for it requires only matrix products and the singular value decomposition of a $k \times k$ matrix.

Applied at first in multifactorial analysis [1], shape analysis [2] and geodesy [3], [4], in the last decade Procrustes analysis was also used to solve some classical photogrammetric problems [5], [6], [7]. In this context, where functional models are non-linear, it proved to be powerful since it does not need $a$ priori approximate parameters values.

Given a matrix $P$ (origin) and a matrix $S$ (destination), containing the coordinates of $n$ points in $\mathbb{R}^{k}$, classical LS Procrustes solutions find the parameters transformation between the two point-sets assuming that all random errors are confined to the destination matrix $S$, whereas $P$ is noisefree. However, this assumption is often unrealistic, since both $P$ and $S$ are corrupted by errors if they derive from measurements. Therefore, it seems appropriate to introduce the Errors-In-Variables (EIV) model, which is a more general model wherein both matrices $P$ and $S$ are assumed to be contaminated by errors. The problem of parameters estimation in the EIV model is often called the total least squares (TLS) problem [8]. Arun [9] showed that for rigid transformations the classical LS orthogonal Procrustes solution coincides with

All the authors are with the DPIA - University of Udine, Via Delle Scienze, 208 - 33100 Udine, Italy (e-mail: maset.eleonora@spes.uniud.it; (fabio.crosilla; andrea.fusiello)@uniud.it) the TLS one. Felus and Burtch [10] instead started from a EIV extended orthogonal Procrustes model to compute the unknown parameters of a similarity transformation (rotation matrix, translation vector and scale factor), showing that, in this case, LS and TLS solution are not coincident.

Recently, an anisotropic variant of the extended orthogonal Procrustes analysis (AEOPA) was developed to solve the exterior orientation problem of one image [6]. This particular version generalizes the extended orthogonal Procrustes model by the fact that the isotropic scale factor is substituted by an anisotropic scaling, i.e., each measurement may have a different scaling factor. The main contribution of this paper is the derivation of a new TLS solution of the AEOPA, assuming that both matrices $P$ and $S$ are affected by random errors.

The paper is organized as follows: in the next section a review of some applied EIV models and their solution is furnished. In Section III the novel TLS Procrustes solutions are described, with particular attention to the anisotropic case. In Section IV the proposed TLS solution of the AEOPA is presented to solve the exterior orientation of an image and compared with the ordinary least squares AEOPA solution [6]. Finally, results and conclusions are discussed in Section V.

\section{ERrors-In-VAriables Model And Total Least SQUARES SOLUTIONS}

The first TLS solutions were developed in literature to solve linear problems expressed by the following EIV model:

$$
\mathbf{y}+\mathbf{e}_{\mathbf{y}}=\left(A+E_{A}\right) \mathbf{x}
$$

where $\mathbf{e}_{\mathbf{y}}$ and $E_{A}$ are the error vector of observations $\mathbf{y}$ and the error matrix of the design matrix $A$, respectively, and $\mathbf{x}$ is the vector of unknown parameters. Golub and Van Loan [8] introduced in mathematical literature the TLS method to treat regression problems where all the data are affected by random errors. The solution they proposed was based on a SVD approach.

Geoinformatics is a field in which EIV models have been applied with success. Felus and Schaffrin [11] developed a Structured Total Least Squares algorithm to solve a planar linear conformal transformation with a particularly structured coefficient matrix $A$. Moreover, they proposed [12] a method based on the non-linear Euler-Lagrange condition equations for estimating a planar affine transformation by a multivariate TLS problem. Results showed that the differences between the TLS and the classical LS estimated parameters are small [12]; nevertheless they could affect significantly the final accuracy of the transformed coordinates. TLS solutions for the 3D datum and affine transformations are also derived in [13] and [14], respectively. 
Furthermore, constraints on the unknown parameters have been often introduced in geodetic applications. Schaffrin [15] solved a TLS problem with linear constraints, whereas the TLS solution of [16] can be applied with arbitrary constraints.

Arun [9] was the first to deal with the TLS solution of the orthogonal Procrustes problem. In the ordinary LS form, the extended orthogonal Procrustes analysis (EOPA) allows to directly estimate the unknown rotation matrix $R(k \times k)$, the translation vector $\mathbf{c}(k \times 1)$ and the global scale factor $\zeta$, for the following model (where $1=[1 \ldots 1]^{\top}$ ):

$$
S-E_{S}=\zeta P R+\mathbf{1} \mathbf{c}^{\top}
$$

for which the square of the Frobenius norm of the residual matrix $E_{S}$ is minimum, i.e.

$$
\min \left\|E_{S}\right\|_{F}^{2}=\min \operatorname{tr}\left[\left(E_{S} E_{S}^{\top}\right)\right]
$$

under the orthogonality condition $R^{\top} R=R R^{\top}=I$.

Replacing model (2) by the EIV model

$$
S-E_{S}=\zeta\left(P-E_{P}\right) R+\mathbf{1} \mathbf{c}^{\top}
$$

where matrix $E_{P}$ represents the errors affecting the coordinates contained in matrix $P$, the unknown $R$, $\mathbf{c}$ and $\zeta$ are then computed by minimizing the square of the Frobenius norm of the matrix $\left[E_{P} \mid E_{S}\right]$, i.e.

$$
\min \left(\left\|E_{P}\right\|_{F}^{2}+\left\|E_{S}\right\|_{F}^{2}\right)=\min \operatorname{tr}\left(E_{P} E_{P}^{\mathrm{\top}}\right)+\operatorname{tr}\left(E_{S} E_{S}^{\mathrm{\top}}\right)
$$

under the constraint that matrix $R$ is orthogonal.

Arun [9] showed that for rigid transformations $(\zeta=1)$ the Procrustes solution of problems (2) and (4) leads to the same result for the unknown parameters $R$ and $\mathbf{c}$.

On the contrary, for similarity transformations the equivalence between the LS solution of model (2) and the TLS solution of the EIV model (4) is disturbed by the scale factor $\zeta$, as demonstrated in [10]. Both methods lead to the following solution for $R$ and $\mathbf{c}$ :

$$
R=U V^{\top}
$$

where $T=U D V^{\top}$ is the SVD of $T$, with $T=\bar{P}^{\top} S$ and $\bar{P}^{\top}=$ $P^{\top}\left(I-\mathbf{1 1}^{\top} /\left(\mathbf{1}^{\top} \mathbf{1}\right)\right)$ is the transposed barycentric matrix $P$,

$$
\mathbf{c}=\left(S^{\top}-\zeta R^{\top} P^{\top}\right) \frac{\mathbf{1}}{\mathbf{1}^{\top} \mathbf{1}} .
$$

However, whereas in the LS case the scale factor is computed from

$$
\operatorname{tr}\left(\bar{P}^{\top} P\right) \zeta-\operatorname{tr}\left(\bar{S}^{\top} P R\right)=0
$$

with $\bar{S}^{\top}=S^{\top}\left(I-\mathbf{1 1}^{\top} /\left(\mathbf{1}^{\top} \mathbf{1}\right)\right)$, the TLS approach leads to the following equation

$$
\operatorname{tr}\left(\bar{S}^{\top} P R\right) \zeta^{2}+\operatorname{tr}\left(\bar{P}^{\top} P-\bar{S}^{\top} S\right) \zeta-\operatorname{tr}\left(\bar{S}^{\top} P R\right)=0
$$

The positive solution of (9) is the searched value of the scale factor. One can notice that (9) contains two out of four terms of (8).

\section{New Procrustes EIV MOdELS}

As previously reported, Felus and Burtch [10] introduced the EIV model in the EOPA problem, obtaining a TLS solution for a similarity transformation. Their method can deal also with the weighting by rows of the coordinate matrices, i.e., to each point, whose coordinates are known in two reference systems, a different weight is given. In this section, we will present an improved version of their algorithm, that admits a different weighting of the two coordinate matrices. The TLS approach can be further extended to the anisotropic orthogonal Procrustes analysis (AEOPA) with row scaling, whose solution is derived in Section III-B and represents the main contribution of this paper.

\section{A. EIV-Weighted Extended Orthogonal Procrustes Analysis (EIV-WEOPA)}

Coordinate matrix can be weighted by columns, that corresponds to assigning a different accuracy to the point coordinate components. Starting from the EIV model (4), the condition (5) to minimize in the weighted case becomes

$$
\min \operatorname{tr}\left(\left[E_{P} \mid E_{S}\right] W_{C} W_{C}^{\top}\left[\begin{array}{c}
E_{P}^{\top} \\
E_{S}^{\top}
\end{array}\right]\right)
$$

where $W_{C}$ is a $(2 k \times 2 k)$ diagonal matrix able to differently weigh the various components. In this case, if $W_{C} \neq I$, no algebraic direct solutions are known in the literature [4], [17], [18]; an iterative solution has been proposed in [18].

However, if we are not interested in weighting differently each component of $P$ and $S$, but we want to assign a different weight to the entries of $P$ versus those of $S$ as a whole, assuming that the two sets $P$ and $S$ have been measured with different accuracy, a closed-form solution can be derived.

Let $1 / \alpha$ and $1 / \beta$ be the weights assigned to $P$ and $S$, respectively. Matrix $W_{C}$ can then be written as

$$
W_{C}=\left[\begin{array}{cc}
\alpha^{-1} I & 0 \\
0 & \beta^{-1} I
\end{array}\right] .
$$

Substituting (11) in (10), it results

$$
\min \operatorname{tr}\left(\left[\alpha^{-1} E_{P} \mid \beta^{-1} E_{S}\right] I I^{\top}\left[\begin{array}{c}
\alpha^{-1} E_{P}^{\top} \\
\beta^{-1} E_{S}^{\top}
\end{array}\right]\right)
$$

under the orthogonality condition $R R^{\top}=R^{\top} R=I$. As described in [9], condition (12) can be rewritten substituting the expression for $\left[E_{P} \mid E_{S}\right]$ computed as the minimum norm solution of (4). After some manipulations, the cost function writes

$$
\begin{aligned}
F= & \operatorname{tr}\left[( \frac { 1 } { \zeta ^ { 2 } + 1 } ) ^ { 2 } ( \frac { \zeta ^ { 2 } } { \alpha ^ { 2 } } + \frac { 1 } { \beta ^ { 2 } } ) \left(\zeta^{2} R^{\top} P^{\top} P R+S^{\top} S+\mathbf{c} \mathbf{1}^{\top} \mathbf{1} \mathbf{c}^{\top}\right.\right. \\
& \left.\left.+2 \zeta R^{\top} P^{\top} \mathbf{1} \mathbf{c}^{\top}-2 S^{\top} \mathbf{1} \mathbf{c}^{\top}-2 \zeta S^{\top} P R\right)+L R^{\top} R-L\right]
\end{aligned}
$$

where $L$ is the matrix of Lagrangian multipliers. The translation vector $\mathbf{c}$ derives from equation

$$
\frac{\partial F}{\partial \mathbf{c}}=\frac{\left(\zeta^{2} / \alpha^{2}+1 / \beta^{2}\right)}{\left(\zeta^{2}+1\right)^{2}}\left[2 \mathbf{1}^{\top} \mathbf{1} \mathbf{c}+2 \zeta R^{\top} P^{\top} \mathbf{1}-2 S^{\top} \mathbf{1}\right]=0
$$


hence

$$
\mathbf{c}=\left(S^{\top}-\zeta R^{\top} P^{\top}\right) \frac{\mathbf{1}}{\mathbf{1}^{\top} \mathbf{1}} .
$$

Setting to zero the derivative of $F$ with respect to $R$, it results

$\frac{\partial F}{\partial R}=0$

$=\frac{\left(\zeta^{2} / \alpha^{2}+1 / \beta^{2}\right)}{\left(\zeta^{2}+1\right)^{2}}\left[2 \zeta^{2} P^{\top} P R+2 \zeta P^{\top} \mathbf{c} \mathbf{1}^{\top}-2 \zeta P^{\top} S\right]+R\left(L+L^{\top}\right)$.

Rotation matrix $R$ can then be computed as

$$
R=U V^{\top}
$$

where $T=U D V^{\top}$ is the SVD of $T$, with $T=\bar{P}^{\top} S$. Substituting (15) in (13), it results

$F=\operatorname{tr}\left[\frac{\left(\zeta^{2} / \alpha^{2}+1 / \beta^{2}\right)}{\left(\zeta^{2}+1\right)^{2}}\left(\zeta^{2} \bar{P}^{\top} P+\bar{S}^{\top} S-2 \zeta \bar{S}^{\top} P R\right)+L R^{\top} R-L\right]$.

The solution for the scale factor $\zeta$ can be obtained by setting to zero the partial derivatives of (18) with respect to $\zeta$, which leads to the following equation

$$
\begin{aligned}
& \frac{2}{\alpha^{2}} \operatorname{tr}\left(\bar{S}^{\top} P R\right) \zeta^{4} \\
& +\left[\frac{4}{\alpha^{2}} \operatorname{tr}\left(\bar{P}^{\top} P\right)-\frac{2}{\beta^{2}} \operatorname{tr}\left(\bar{P}^{\top} P\right)-\frac{2}{\alpha^{2}} \operatorname{tr}\left(\bar{S}^{\top} S\right)\right] \zeta^{3} \\
& +\left[-\frac{6}{\alpha^{2}} \operatorname{tr}\left(\bar{S}^{\top} P R\right)+\frac{6}{\beta^{2}} \operatorname{tr}\left(\bar{S}^{\top} P R\right)\right] \zeta^{2} \\
& +\left[\frac{2}{\alpha^{2}} \operatorname{tr}\left(\bar{P}^{\top} P\right)+\frac{2}{\beta^{2}} \operatorname{tr}\left(\bar{P}^{\top} P\right)-\frac{4}{\beta^{2}} \operatorname{tr}\left(\bar{S}^{\top} S\right)\right] \zeta \\
& -\frac{2}{\beta^{2}} \operatorname{tr}\left(\bar{S}^{\top} P R\right)=0 .
\end{aligned}
$$

The searched value of $\zeta$ is the root of the $4^{\text {th }}$ order polynomial (19) that minimizes the cost function (18).

\section{B. EIV-Anisotropic Orthogonal Procrustes Analysis (EIV- AEOPA)}

The EOPA model (2) can be generalized substituting the isotropic scale factor $\zeta$ with an anisotropic scaling characterized by a diagonal matrix $Z=\operatorname{diag}\left(\zeta_{1} \ldots \zeta_{n}\right)$ of different scale values:

$$
S-E_{S}=Z P R+\mathbf{1} \mathbf{c}^{\top} .
$$

The LS solution of this problem, known as AEOPA with row scaling [17], can be obtained with the Lagrange multipliers method as follows.

Defining the Lagrangian function

$$
F\left(E_{S}, L, R, \mathbf{c}, Z\right)=\operatorname{tr}\left(E_{S} E_{S}^{\top}\right)+\operatorname{tr}\left[L\left(R^{\top} R-I\right)\right]
$$

where $L$ is the matrix of Lagrangian multipliers and setting to zero the partial derivatives with respect to the unknowns $R, \mathbf{c}$ and $Z$, one obtains the following solving equations:

$$
\begin{gathered}
R=U V^{\top} \text { with } U D V^{\top}=P^{\top} Z\left(I-\mathbf{1} \mathbf{1}^{\top} / n\right) S \\
\mathbf{c}=(S-Z P R)^{\top} \mathbf{1} / n \\
\left(Z A A^{\top}-B A^{\top}\right) \odot I=0
\end{gathered}
$$

where $A=P R, B=S-\mathbf{1} \mathbf{c}^{\top}$ and $\odot$ is the Hadamard (or element-wise) product.

Whereas in the solution of the EOPA problem one can recover first $R$, that does not depend on the other unknowns, then the isotropic scale factor $\zeta$, and finally $\mathbf{c}$, in the anisotropic case the unknowns are entangled in such a way that there is no direct solution available. An iterative procedure, the so called block relaxation scheme, is then needed, where each variable is alternatively estimated while keeping the others fixed.

The solution given by [6] assumes that all random errors are confined to the destination matrix $S$. However, even in the anisotropic case it appears more realistic to take into account also the errors affecting matrix $P$. Model (20) can therefore be substituted by the following EIV model, that we will henceforth call EIV-AEOPA model:

$$
S-E_{S}=Z\left(P-E_{P}\right) R+\mathbf{1} \mathbf{c}^{\top} .
$$

A TLS estimation of the parameters of this model has never been developed in the literature, hence we derive its solution below. Following the traditional Lagrangian approach, the target function to be minimized can be written as

$$
\begin{aligned}
& F\left(E_{S}, E_{P}, G, L, \mathbf{c}, R, Z\right)=\frac{1}{\alpha} \operatorname{tr}\left(E_{S} E_{S}^{\boldsymbol{\top}}\right)+\frac{1}{\beta} \operatorname{tr}\left(E_{P} E_{P}^{\boldsymbol{\top}}\right)+ \\
& \quad 2 \operatorname{tr}\left[G^{\boldsymbol{\top}}\left(S-E_{S}-Z P R+Z E_{P} R-\mathbf{1} \mathbf{c}^{\top}\right)\right]+ \\
& \quad 2 \operatorname{tr}\left[L\left(R^{\top} R-I\right)\right]
\end{aligned}
$$

where $G$ and $L$ are the matrix of Lagrangian multipliers, whereas $1 / \alpha$ and $1 / \beta$ are different weights assigned to $S$ and $P$, respectively. This can be solved by setting to zero the partial derivatives with respect to the unknowns. Eventually, the following necessary equations can be obtained:

$$
\begin{aligned}
\frac{\partial F}{\partial E_{S}} & =\frac{2}{\alpha} E_{S}-2 G=0 \\
\frac{\partial F}{\partial E_{P}} & =\frac{2}{\beta} E_{P}+2 Z^{\top} G R^{\top}=0 \\
\frac{\partial F}{\partial G} & =2\left(S-E_{S}-Z P R+Z E_{P} R-\mathbf{1} \mathbf{c}^{\top}\right)=0 \\
\frac{\partial F}{\partial L} & =2\left(R^{\top} R-I\right)=0 \\
\frac{\partial F}{\partial \mathbf{c}} & =-2 G^{\top} \mathbf{1}=0 \\
\frac{\partial F}{\partial R} & =2\left(E^{\top} Z^{\top}-P^{\top} Z^{\top}\right) G+2 R\left(L^{\top}+L\right)=0 \\
\frac{\partial F}{\partial Z} & =G\left(R^{\top} E^{\top}-R^{\top} P^{\top}\right) \odot I=0
\end{aligned}
$$

The translation vector c can be computed from (27), (28), (29) and (32):

$$
\mathbf{c}=(S-Z P R)^{\top}\left(\alpha I+\beta Z^{2}\right)^{-1} \frac{\mathbf{1}}{\varepsilon}
$$

where $Z^{2}=Z Z^{\top}$ and $\varepsilon=\mathbf{1}^{\top}\left(\alpha I+\beta Z^{2}\right)^{-1} \mathbf{1}=\operatorname{tr}\left[\left(\alpha I+\beta Z^{2}\right)^{-1}\right]$.

Equations (28), (29) and (31) give:

$$
R=U V^{\top}
$$


where $T=U D V^{\top}$ is the SVD of $T$, with

$$
T=P^{\top} Z^{\top}\left(\alpha I+\beta Z^{2}\right)^{-1}\left[I-\frac{\mathbf{1 1}^{\top}}{\varepsilon}\left(\alpha I+\beta Z^{2}\right)^{-1}\right] S
$$

Finally, combining (27), (28), (29) and (33) one obtains:

$$
\left(\beta Z A B^{\top} Z+\alpha Z A A^{\top}-\beta B B^{\top} Z-\alpha B A^{\top}\right) \odot I=0
$$

with $A$ and $B$ previously defined. Equation (37) can be solved independently for each element $\zeta_{i}$ of matrix $Z$. The value of each scale factor is retrieved from the following equation:

$$
\begin{array}{r}
\left(\beta \sum_{j=1}^{3} A_{i j} B_{i j}\right) \zeta_{i}^{2}+\left(\alpha \sum_{j=1}^{3} A_{i j}^{2}-\beta \sum_{j=1}^{3} B_{i j}^{2}\right) \zeta_{i}- \\
\alpha\left(\sum_{j=1}^{3} A_{i j} B_{i j}\right)=0
\end{array}
$$

Analyzing (38), it can be noticed that there are two real solutions, one negative and one positive; the latter is the searched value of the scale factor $\zeta_{i}$.

Comparing the solving equations for $Z$ derived from the classical model (20) and from the EIV model (25), it is easy to see that the two terms of the LS solution (24) are contained in the TLS solution (37). This is the same property previously highlighted in the isotropic case. Let us rewrite (24) and (37) as follows:

$$
\begin{gathered}
{\left[(Z A-B) A^{\top}\right] \odot I=0} \\
{\left[\beta(Z A-B) B^{\top} Z+\alpha(Z A-B) A^{\top}\right] \odot I=0 .}
\end{gathered}
$$

One can notice that, if the system $Z A=B$ has an exact solution (i.e., matrices $P$ and $S$ are error-less), this satisfies both (39) and (40). Therefore in the ideal case ordinary LS and TLS approaches coincide, as expected, whereas they lead to different solutions when the data are affected by noise.

Similarly to the LS solution of the AEOPA problem, the TLS solution of the EIV-AEOPA model requires a block relaxation scheme to estimate the unknowns $R, \mathbf{c}$ and $Z$.

\section{EXPERIMENTAL VALIDATION}

In the paper of Garro et al. [6] the AEOPA with row scaling was applied to the problem of estimating the position and orientation of a perspective camera given its intrinsic parameters and a set of world-to-image correspondences, known as exterior orientation problem in Photogrammetry or Perspective-n-Point camera pose in Computer Vision.

We briefly review here how this problem can be formulated in terms of model (20) or (25). Given at least three control points and their projections, the exterior orientation problem requires to find a rotation matrix $R$ and a vector $\mathbf{c}$ (specifying attitude and position of the camera) such that the vector form of collinearity equations:

$$
\mathbf{p}_{i}=\zeta_{i}^{-1} R\left(\mathbf{s}_{i}-\mathbf{c}\right)
$$

is satisfied for some positive scalar $\zeta_{i}$, where

- $\mathbf{s}_{i}$ is the coordinate vector of the $i$-th control point in the external system;
- $\mathbf{c}$ is the coordinate vector of the projection center in the external system;

- $\zeta_{i}$ is a positive scalar proportional to the "depth" of the point, i.e., the distance from the $i$-th control point to the plane containing the projection center and parallel to the image plane;

- $R$ is the rotation matrix transforming from the external system to the camera system;

- $\mathbf{p}_{i}$ is the coordinate vector of the $i$-th control point in the camera system, where the third component is equal to $-c$, the principal distance or focal length.

Expressing (41) with respect to $\mathbf{s}_{i}$ yields:

$$
\mathbf{s}_{i}=\zeta_{i} R^{\top} \mathbf{p}_{i}+\mathbf{c} .
$$

After transposing and extending to $n$ control points $\mathbf{s}_{1} \ldots \mathbf{s}_{n}$, it results:

$$
S=Z P R+\mathbf{1} \mathbf{c}^{\top}
$$

where $P$ is the matrix by rows of image point coordinates defined in the camera frame, $S$ is the matrix by rows of point coordinates defined in the external system, $Z$ is the diagonal (positive) depth matrix.

Formula (43) can be rewritten in the form of the AEOPA model (20) or the EIV-AEOPA model (25), according to whether the error is assumed to affect only $S$ or both $S$ and $P$. It is particularly significant in this photogrammetric application the capability of our solution (26) to take into account the different variances of $E_{P}$ and $E_{S}$, since image coordinate $\mathbf{p}_{i}$ and $3 \mathrm{~d}$ points coordinate $\mathbf{s}_{i}$ are measured with different accuracy.

Garro et al. [6] compared the LS solution of (20) with state of the art algorithms that perform the exterior orientation, showing that AEOPA reaches the best trade-off between speed and accuracy. In this paper, we tested the proposed TLS solution of the AEOPA for solving the exterior orientation of an image against the LS formulation [6].

To carry out the simulation, $n=\{6, \ldots, 30\} 3 \mathrm{D}$ points were randomly distributed in a sphere of unit radius centered on the origin and perturbed with random noise with standard deviation $\sigma_{S}=\{0.10,0.27,0.71,1.88,5.00\}[\mathrm{mm}]$ (corresponding to five logarithmically spaced values from 0.10 to 5.00 ). The camera was positioned at distances of 5 and 10 meters from the origin and the focal length was chosen so as to yield a view angle of $60^{\circ}$ with an image size of $1000 \times 1000$ pixels. Different values of noise $\sigma_{P}=\{1, \ldots, 5\}$ [pixel] were added to the image coordinates obtained from the projection of the noise free 3D points. For each setting the test was run 500 times and the mean and median error norms were computed. In all the experiments the initial depths were set to one. Results are reported in Fig. 1 and 2. As a figure of merit only the rotation errors are shown, since the behavior of the translations errors is similar. The rotation error is the angle of the residual rotation, computed as $\left\|\log \left(R^{\mathrm{T}} \hat{R}\right)\right\|_{F}$, where $R$ is the ground truth, $\hat{R}$ is the actual rotation and $\|\cdot\|_{F}$ is the Frobenius norm.

Comparing the median error, one can notice that EIVAEOPA and AEOPA lead almost to the same accuracy. On the other hand, the root mean square error (RMSE) is different when the number of correspondences in the image is small 
$(n \leq 15)$ (this is clear in Figure 1 where 10 points are considered). This is due to the fact that AEOPA in a few cases converges to wrong results, that are not sufficient to skew the median, but affects the mean error. The rundown of this simulation is that the new TLS solution works better than the ordinary LS algorithm when the number of reference points (whose coordinate are known in both the camera and the external reference frame) is small, which is a common situation in Photogrammetry.

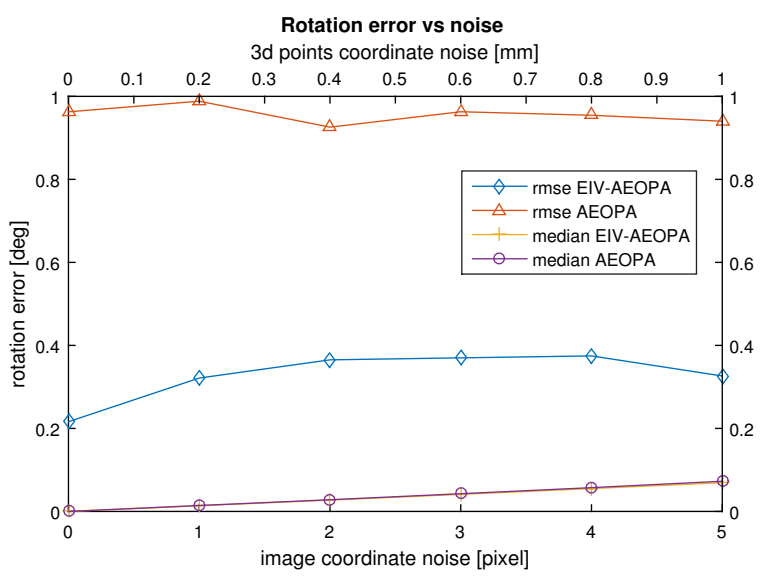

Fig. 1. Rotation error vs noise using 10 correspondences and a distance of the camera from the origin equals to $10 \mathrm{~m}$. The RMSE and the median rotation errors are plotted against the standard deviation of the noise added to image coordinates and $3 \mathrm{~d}$ points coordinate.

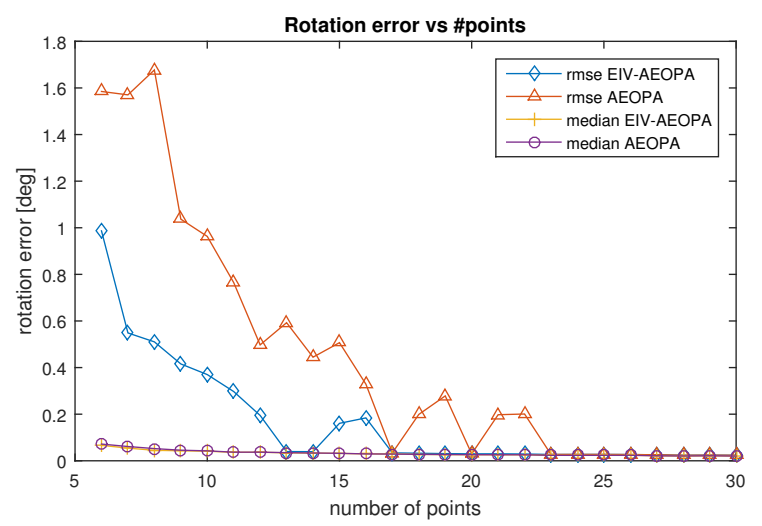

Fig. 2. Rotation error vs number of points. The RMSE and the median rotation errors are plotted against the number of points that have been used. The distance of the camera from the origin is equal to $10 \mathrm{~m}$ and the standard deviation of the noise added is 3 pixel for the image coordinate and $0.71 \mathrm{~mm}$ for the $3 \mathrm{~d}$ points coordinate.

\section{CONCLUSION}

In this paper, a novel total least squares solution of the AEOPA with row scaling has been proposed. The analytic model considers that both the origin and destination points coordinates are affected by random errors (EIV model). In this way, an algorithm that maintains all the advantages of the classical Procrustes analysis was obtained. In particular, the problems related to linearization and approximate parameters values determination were avoided. At the same time, the unrealistic assumption that the source coordinates are errorless was removed. This Errors-In-Variables model is closer to reality and leads to a more realistic estimation of the unknown transformation parameters.

The developed TLS solution of the AEOPA problem was tested for solving the exterior orientation of an image. Experiments show that when the number of reference points, whose coordinate are known in both the camera and the external frame, is small, the proposed total least squares method leads to an accuracy in the parameters estimation that is higher than the one reached with the ordinary least squares anisotropic Procrustes solution.

The proposed EIV-AEOPA algorithm can be a valuable tool in other computer vision and photogrammetric problems. In a future work, its application for solving bundle block adjustment will be investigated.

\section{REFERENCES}

[1] B. F. Green, "The orthogonal approximation of an oblique structure in factor analysis," Psychometrika, vol. 17, no. 4, pp. 429-440, 1952.

[2] F. L. Bookstein, "Size and shape spaces for landmark data in two dimensions," Statistical Science, vol. 1, no. 2, pp. 181-222, 1986.

[3] F. Crosilla, "Procrustean transformation as a tool for the construction of a criterion matrix for control networks," Manuscripta geodetica, vol. 8, 1983.

[4] _- "Procrustes analysis and geodetic sciences," in Quo vadis geodesia...? Department of Geodesy and GeoInformatics, University of Stuttgart, 1999, vol. 1, pp. 69-78.

[5] A. Beinat, F. Crosilla, and D. Visintini, "Examples of georeferenced data transformations in GIS and digital photogrammetry by procrustes analysis techniques," The International Archives of Photogrammetry and Remote Sensing, vol. XXXII, 6W8/2, 2000.

[6] V. Garro, F. Crosilla, and A. Fusiello, "Solving the pnp problem with anisotropic orthogonal procrustes analysis," in Second Joint 3DIM/3DPVT Conference: 3D Imaging, Modeling, Processing, Visualization and Transmission, 2012.

[7] A. Fusiello and F. Crosilla, "Solving bundle block adjustment by generalized anisotropic procrustes analysis," ISPRS Journal of Photogrammetry and Remote Sensing, vol. 102, pp. 209-221, April 2015.

[8] G. H. Golub and C. F. Van Loan, "An analysis of the total least squares problem," SIAM Journal on Numerical Analysis, vol. 17, no. 2, pp. 883 893, 1980.

[9] K. S. Arun, "A unitarily constrained total least squares problem in signal processing," SIAM Journal on Matrix Analysis and Applications, vol. 13, no. 3, pp. 729-745, 1992.

[10] Y. A. Felus and R. C. Burtch, "On symmetrical three-dimensional datum conversion," GPS Solutions, vol. 13, no. 1, pp. 65-74, 2009.

[11] Y. Felus and B. Schaffrin, "Performing similarity transformations using the errors-in-variables-model," in Proceedings of the ASPRS Meeting, Baltimore/MD, 2005.

[12] B. Schaffrin and Y. Felus, "On the multivariate total leastsquares approach to empirical coordinate transformations. three algorithms," Journal of Geodesy, vol. 82, no. 6, pp. 415-421, 2008.

[13] B. Li, Y. Shen, and W. Li, "The seamless model for three-dimensional datum transformation," Science China Earth Sciences, vol. 55, no. 12, pp. 2099-2108, 2012

[14] B. Li, Y. Shen, X. Zhang, C. Li, and L. Lou, "Seamless multivariate affine error-in-variables transformation and its application to map rectification," International Journal of Geographical Information Science, vol. 27, no. 8, pp. 1572-1592, 2013.

[15] B. Schaffrin, "A note on constrained total least-squares estimation," Linear Alg. Appl., vol. 417, pp. 245-258, 2006.

[16] X. Fang, "Weighted total least-squares with constraints: a universal formula for geodetic symmetrical transformations," Journal of Geodesy, vol. 89, no. 5, pp. 459-469, 2015.

[17] J. C. Gower and G. B. Dijksterhuis, Procrustes problems, ser. Oxford Statistical Science Series. Oxford University Press, 2004.

[18] M. A. Koschat and D. F. Swayne, "A weighted procrustes criterion," Psychometrika, vol. 56, no. 2, pp. 229-239, 1991. 